\title{
Is Time Misconception of the Transformations Possible?
}

\author{
Mesut KAVAK
}

Time is quite interesting phenomenon in physics, and it seems is relative; but what does it mean to be relative of time? What does to be fixed of light speed mean? Does to be fixed of light speed require observation at light speed? What if we can observe faster than light speed because of increased frame number? Is time effective for this imaginary effect or also time itself is dependent on another actual causative phenomenon? Is it possible to make a wrong conception for time and speed even if the phenomenon we advocate is actually true?

\section{Introduction}

Distribution of image at light speed brings some other phenomena out together with itself. Namely, light speed cannot change even if you take a relative speed like $2 c$ which is bigger than $c$. It does not change due to observer, it changes due to observer as illusion. It is always fixed; but each frame of the transfer can change being the light speed or transfer speed is fixed. Namely while you are getting closer to an object which is moving in any direction, you detect it faster than its actual speed if you move towards it, and also the observed object gets bigger; but it has an end since the distance between you and it is limited. Namely when you get the closest distance, the relative acceleration converges to zero; because the history of the observed object is stored between you and the object according to the distance between you and the object, and you can watch its history up to $2 \mathrm{x}$ faster whatever the actual direction and speed of the object are, if you can go at speed of light towards the object.

\section{A compatible transformation}

The below stated formulas are for scalar magnitudes. You must redetermine their marks according to direction of moving bodies.

Warning

There are 3 points which are on the same line and are going to be used for something for motional transformations on Fig. 1.

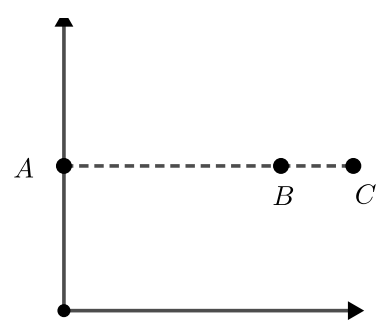

Fig. 1: 3 points in space on the same line

Here we should not intermingle outer space speed of objects and its observational transfer speed. Image transferring speed is light speed and can get any value for different universe being fixed. We accept it as $c$.

Now think that $A$ and $C$ are fixed. Naturally $C$ observes $A$ as fixed; but there is a special condition that is valid for any universe, $C$ observes the old condition of $A$ in changing times according to the distance between them; because light speed is limited. Even, if $A$ and the distance between $A$ and $C$ are big enough, for example when $A$ has already been disappeared by losing its all energy somehow, $C$ still observes it since there is a time difference.

\subsection{Constant speed motion}

Now think that $C$ is moving towards $A$ on the same line being $A$ is fixed or $A$ is moving towards $C$ on the same line being $C$ is fixed or they both move towards each other. As we always observe image of any object, we can write it as Eq. (1) and Eq. (2).

$$
\begin{aligned}
& v_{A}^{\prime}=\left(\frac{c+v_{C}}{c}\right) v_{A} \\
& v_{C}^{\prime}=\left(\frac{c+v_{A}}{c}\right) v_{C}
\end{aligned}
$$

Here $v_{A}^{\prime}$ and $v_{C}^{\prime}$ are observed speed of $A$ and $C .(c+v) / c$ is a multiplier according to initial speeds of moving objects. As we always observe the history of objects by some frames, this is increase amount of the observed frame number in an unit of time over actual and simultaneous speed of observed object that this speed had gained somehow over $F t=m v$ work or over any function without reference. Here the thing which increases the relative speed of $A$ is the speed of $C$ since $C$ is going to experience more frame. Also the thing which increases the relative speed of $C$ is the speed of $A$ since $A$ is going to experience more frame as well.

As it can be seen over Eq. (1) and Eq. (2), if there are some objects which the sum of their speed as vector quantity is different than zero, then they experience different times relatively.

\section{Inference}

Frame number is partially dependent on and actually is independent of light speed. Whatever the initial velocities of objects are, image is distributed at light speed; so if it is possible to move towards an object faster then light by any multiples of $c$ as $n c$, you detect the observed object as much more faster due to $n c$. As long as you get faster, you detect the object faster and faster; but as also you cannot move faster than light, you cannot detect objects faster than a limit. Limit of both frame number and the speed of the object which its image is transferred are naturally dependent on light speed by this way.

Right this point you can ask, that for example while $C$ is observing $A$, the initial speed of $C$ changes the observed object's actual outer speed which emerges according to a global time and gained by $F t=m v$, changes by $\left(c+v_{C}\right) / c$; but at that time is not $v_{A}$ is inclusive to show itself at higher speed by increasing its own initial speed? The answer is exact no; because this is an observation and cannot be realized from two points at the same time. Namely, even if $v_{A}$ increases at that time constantly, the observer detects its acceleration after required time according to its own speed but not more. The 
observer detects a resulting acceleration whatever happened there. As a result, you cannot include a new ratio by $\left(c+v_{A}\right) / c$ to the observer formula.

The universe emerges over time since has limited energy. It gains its total energy for 1 second. It means there are time differences for each point of space. Namely each cubic meter has time difference by $1 / V$ where $V$ is volume of the universe; so when I said global time, it means when that point has $E_{1}$ and the other has $E_{2}$ energy. Namely still each point of space get different time according to this global reference time; but as the amount is too small, we should not include it to these calculations. Time is absolute by these rules. The difference between global time of $t$ and $t^{\prime}$ is to be observational time of $t^{\prime}$. Namely all events occur in 1 second by ignored time differences due to the global time; but you cannot observe all of them as also you cannot be observed. This depends due to the distance between observation elements.

\section{Warning}

Think that $n$ times particle pass from a point at light speed. If point starts to move in the direction of particles at $v$ speed, to see how many particles are detected for $c+v$, the equation becomes $n(c+v) / c$. Here $n$ can be speed of the observed object, and $v$ can be speed of the observer. Even if the point has an initial speed, you cannot make a fiction such as if $n$ times particle pass at $c+v_{i}$, then for $c+v_{i}+v_{2}$, there $n\left(c+v_{i}+\right.$ $\left.v_{2}\right) /\left(c+v_{i}\right)$ times particle are detected. Image transfer speed is always fixed as light speed. You always see each frame at light speed; but as you have an initial speed, you detect it like it increased since the energy collected increases in an unite of time. Namely for initial speed, you must change either $n$ or if you accept $n$ as still fixed, it means the observed object is slower.

You cannot accept one of the moving bodies as static to calculate speed over the other one of them. This changes everything. Namely, for two bodies move towards each other, you cannot make an addition like $v_{A}+v_{C}=v_{A}^{\prime}=v_{C}^{\prime}$ operation. Time passes different for these if they move, and speeds are detected different as relatively. Time does not change in the same ratio. Such an addition can only be made if one of them is really static according to global time.

\section{Warning}

To calculate actual independent global position of objects, first we must fix time which is detected different because of observation speed.

$$
\begin{aligned}
& x_{A}^{\prime}(t)=v_{A}^{\prime} t \\
& x_{C}^{\prime}(t)=v_{C}^{\prime} t
\end{aligned}
$$

Eq. (3) and Eq. (3) are relative positions. $x_{A}^{\prime}$ is the position which is estimated by $C$, and also $x_{C}^{\prime}$ is the position which is estimated by $A$ over the same global $t$; so to fix them, we must fix times first over Eq. (5) and Eq. (6).

$$
\begin{aligned}
& x_{A}(t)=x_{A}^{\prime}(t)+t\left(v_{A}-v_{A}^{\prime}\right) \\
& x_{C}(t)=x_{C}^{\prime}(t)+t\left(v_{C}-v_{C}^{\prime}\right)
\end{aligned}
$$

Now let us use more object to analyze if there are some special conditions. If the three points on Fig. 1 are handled together, we can write $x^{\prime}=x-v_{B} t$ and $t^{\prime}=t$ according to the global time when $A$ and $C$ are fixed but $B$ moves towards to
$C$; but is the formula or only its values change due to observation?

As $A$ and $C$ are fixed, they observe $B$ at its actual speed. There is no time deviation, and thus $x^{\prime}=x-v_{B} t$ is valid for $t^{\prime}=t$.

If $A$ and $B$ move in the direction of $C$, and $C$ moves in the opposite direction being all of them at the same speed. For this condition, total observation of $B$ becomes Eq. (7).

$$
2 v_{B}=\frac{\left(c-v_{A}\right) v_{B}}{c}+\frac{\left(c+v_{C}\right) v_{B}}{c}
$$

It means even if the observation changes due to observer, actually there is no real deviation or delay. This happens for only partial observers which cannot detect all motions at the same time. It signs to a global time. Time is conserved.

\subsection{Acceleration motion}

If we apply the above stated rules to acceleration motion, it becomes Eq. (8) and Eq. (9) over Eq. (1) and Eq. (1),

$$
\begin{aligned}
& a_{A}^{\prime}=\left(\frac{c+a_{C} t}{c}\right) a_{A} \\
& a_{C}^{\prime}=\left(\frac{c+a_{A} t}{c}\right) a_{C}
\end{aligned}
$$

where $t$ is global time. For these values, to calculate actual independent global position of objects, again firstly we must fix time which is detected different because of observation speed.

$$
\begin{aligned}
& x_{A}^{\prime}(t)=a_{A}^{\prime} t^{2} \\
& x_{C}^{\prime}(t)=a_{C}^{\prime} t^{2}
\end{aligned}
$$

Eq. (10) and Eq. (11) are relative positions. $x_{A}^{\prime}$ is the position which is estimated by $C$, and also $x_{C}^{\prime}$ is the position which is estimated by $A$ over the same global $t$; so to fix them, we must fix times first over Eq. (12) and Eq. (13).

$$
\begin{aligned}
& x_{A}(t)=x_{A}^{\prime}(t)+t^{2}\left(a_{A}-a_{A}^{\prime}\right) \\
& x_{C}(t)=x_{C}^{\prime}(t)+t^{2}\left(a_{C}-a_{C}^{\prime}\right)
\end{aligned}
$$

Let us analyze the acceleration condition somehow.

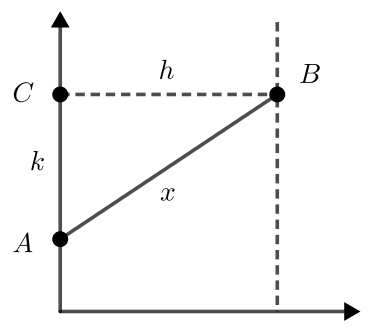

Fig. 2: Satellite

Here on Fig. 2 there is a satellite at $B$ point moves on the parallel line to $y$ axis. $C B=h$ is the height, $C$ is a point which its coordinate is known, $A$ is an object which moves on the $y$ axis. $A B=x$ is the distance between the object and the satellite.

Now assume that $B$ moves in the direction of $+y$ axis and $A$ moves in the direction of $-y$ axis. Namely time slows down for the both relatively and they detect each other as slowing down since they move away from each other, and thus frame number is going to decrease for an unite of time. $k$ is depen- 
dent on the velocities of both $A$ and $B$; so it becomes Eq. (14)

$$
k=\left(v_{A}+v_{B}\right) t
$$

The time here must be the same time, and is the time of acceleration if the speed is not fixed. Also it starts after a calibration for the current values at $t_{0}=0$ moment and $k=0$ point. $x$ becomes for this as

$$
x=\sqrt{\left(v_{A}+v_{B}\right)^{2} t^{2}+h^{2}}
$$

Now the condition has been turned into the condition of a linear motion over linear $x$ distance; but the change emerges over the rule of $x$ as Eq. (15). It means the motion is like acceleration even for the fixed speeds; so it can written as $x=a t^{2}=a_{A} t^{2}+a_{B} t^{2}$ and thus over this as $x / t^{2}=a=a_{A}+a_{B}$ since is not observation yet, and is due to global time as independent. Hence it becomes Eq. (16) over Eq. (8) or Eq. (9),

$$
a_{A}^{\prime}=\left(\frac{c+a_{B} t}{c}\right)\left(a-a_{B}\right)
$$

where $a-a_{B}=a_{A}$ or becomes Eq. (17),

$$
a_{A}^{\prime}=\left(\frac{c+\left(a-a_{A}\right)}{c}\right)\left(a_{A}\right)
$$

where $a-a_{B}=a_{A}$ over the same Eq. (8) or Eq. (9). If they are equalized to each other, it becomes Eq. (18),

$$
a=\frac{a_{B}^{2}(1-t)+2 a_{B} c}{2 c}
$$

and over Eq. (18) it becomes Eq. (19) where $a=a_{A}+a_{B}$.

$$
a_{A}=\frac{a_{B}^{2}(1-t)}{2 c}
$$

As we know $a$ over Eq. (15) as $x / t^{2}$, we can find $a_{B}$ over Eq. (18) and then $a_{A}$ over Eq. (19). Hence, we can find the deviation or actual positions over Eq. (8), Eq. (9) and Eq. (12), Eq. (13).

\section{Energy transformation}

As I proved [1], formation motion in the other name the motion which emerges to create matter and outer space observational motion are accepted together. There is no other motion. When mass moves from A to B in space, it means creation motion which is because of its existence moves from A to B. Matter uses the same space at the same time for any motion and work. For this condition, assume that kinetic energy is dependent of an operator like $N m v^{2}$ that $N$ can be a rational number as also can be an integer. Now total kinetic energy which matter has for its existence as $N m c^{2}$ must be equal to the sum of observational kinetic energy $N m v^{2}$ and static kinetic energy $N m_{0} c^{2}$ in the other name static total energy before gaining observational kinetic energy. For this definition, it becomes Eq. (20).

$$
N m_{0} c^{2}+N m v^{2}=N m c^{2}
$$

For Eq. (20), change in mass becomes Eq. (21).

$$
m=\frac{m_{0}}{1-\frac{v^{2}}{c^{2}}}
$$

This mass is the mass which already had been accelerated and is moving at a constant speed of $v$. For acceleration work, if $p_{0}=m_{0} c$ and $v=F t / m_{0}$, then for $\int m d_{v}$, it will be Eq. (22).

$$
m=p_{0} \tanh ^{-1}\left(\frac{F t}{p_{0}}\right)
$$

On $F t=m v$ equation, the multiplication of $F t$ does not change even if the mass or the velocity on the momentum equation changes by any rule; because they are self-formed according to $F t$ work if this the equation; so over $F t=m v_{r}=$ $m_{0} v$ equation, the actual reached velocity $v_{r}$ will be Eq. (23).

$$
v_{r}=v\left(1-\frac{v^{2}}{c^{2}}\right)
$$

For Eq. (21) and Eq. (23), kinetic energy becomes Eq. (24) over $W=m v_{r}^{2}$

$$
W=m_{0} v^{2}\left(1-\frac{v^{2}}{c^{2}}\right)
$$

Total energy becomes Eq. (25).

$$
E=\frac{m_{0} c^{2}}{1-\frac{v^{2}}{c^{2}}}
$$

As it can be seen over Eq. (25), mass and energy magnitudes are not conserved in focal point even if work done energy is conserved. During this changing, total density of universe changes; but its total energy is always conserved. For the above stated equations it becomes $v^{2} / c^{2}=0$ over $E=W+$ $m_{o} c^{2}$. It means it is always $v=0$ that it is always $c$. This is the verifying. Light speed is the highest speed, and the lower is not possible. Matter uses the same space at the same time.

Now the situation became more interesting; because Eq. (1) and Eq. (2) must turn into Eq. (26) and Eq. (27) over the energy transformation.

$$
\begin{aligned}
& v_{A}^{\prime}=\left(1+\frac{v_{C} c^{2}-v_{C}^{3}}{c^{3}}\right)\left(1-\frac{v_{A}^{2}}{c^{2}}\right) v_{A} \\
& v_{C}^{\prime}=\left(1+\frac{v_{A} c^{2}-v_{A}^{3}}{c^{3}}\right)\left(1-\frac{v_{C}^{2}}{c^{2}}\right) v_{C}
\end{aligned}
$$

For this final condition when you assume that they move towards each other at the same speed, a graph emerges like the below.

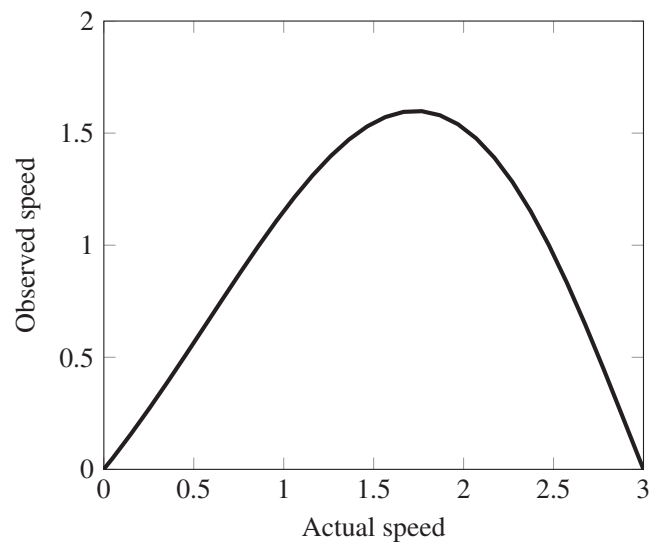

Fig. 3: Observational speed over actual speed where $x=c / 10^{8}$ and the speeds are the same

The results are quite interesting; because as long as you 
get faster, you detect the objects slower; because when you get closer light speed during observational motion not creation that already creation is at light speed, observational ability decreases; because you create an imaginary history in the environment between objects since you get closer to image transfer speed. Also a table emerges like the below.

Table 1: Observational speed change of objects over relativistic energy transformations

\begin{tabular}{c|c|c|c|c}
$v_{A}$ & $\mathrm{c} / 4$ & $\mathrm{c} / 3$ & $\mathrm{c} / 2$ & $3 \mathrm{c} / 4$ \\
\hline$v_{A}^{\prime}$ & $0.290 \mathrm{c}$ & $0.384 \mathrm{c}$ & $0.516 \mathrm{c}$ & $0.436 \mathrm{c}$
\end{tabular}

Your appearance time to existence and image transfer time close to each other since there is a single motion for creation and other space motions; but it is more interesting, after $c / 2$ in the table, again observational speed decreases. The following is the graph of the same rule.

When you assume that they move towards each other being the observer $v_{C}$ has $1 \mathrm{~m} / \mathrm{s}$ speed and the other one has changing speeds, a graph emerges like the below.

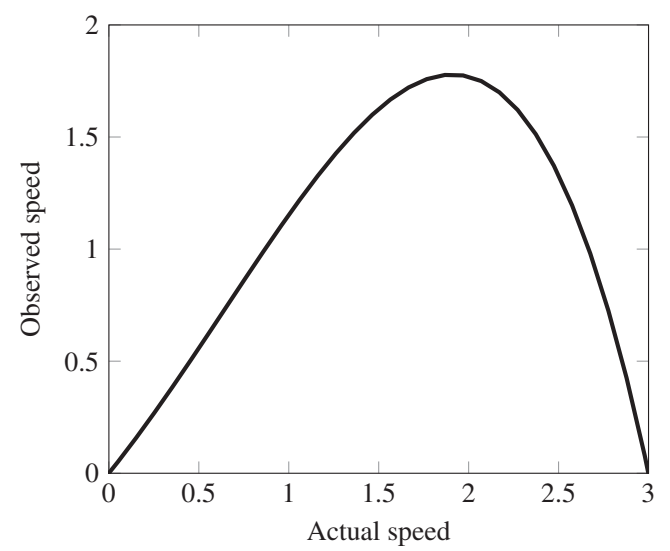

Fig. 4: Observational speed over actual speed where $x=c / 10^{8}$ and the speed of the observer is fixed

In the same manner it is interesting, after $c / 2$ in the table, again observational speed decreases that actually it is not related with $c / 2$ since also starts decrease before it. It seems, always we must accept total speed of the objects as $c$.

Table 2: Another table for almost static objects

\begin{tabular}{c|c|c|c|c}
$v_{A}$ & $\mathrm{c} / 4$ & $\mathrm{c} / 3$ & $\mathrm{c} / 2$ & $3 \mathrm{c} / 4$ \\
\hline$v_{A}^{\prime}$ & $0.234 \mathrm{c}$ & $0.296 \mathrm{c}$ & $0.375 \mathrm{c}$ & 0.328
\end{tabular}

What is going on the earth?

\section{Conclusion}

Here I made some inferences; but I really do not know yet what they mean. Even so it seems that time is relative; but actually this is not against to be absolute of time. There is a single time which is creation time of any bodies in the universe for 1 second. The relativistic time emerges because of distance and image transfer speed. We always observe the history of objects in changing times due to distance; but actually this does not mean a time travel or staying as a young person is possible since is only relativistic effect. Age, entropy, total energy etc. are dependent on global time.

\section{References}

1. Kavak M. 2018, Complementary Inferences on Theoretical Physics and Mathematics, OSF Preprints, Available online: https://osf.io/tw52w/ 